\title{
MicroRNA-149 Increases the Sensitivity of Colorectal Cancer Cells to 5-Fluorouracil by Targeting Forkhead Box Transcription Factor FOXM1
}

\author{
Xiaobei Liua,c Tao Xie ${ }^{b}$ Xiaobei Maoc Lijun Xue ${ }^{c}$ Xiaoyuan Chuc \\ Longbang Chen ${ }^{\mathrm{a}, \mathrm{c}}$
}

\begin{abstract}
aDepartment of Medical Oncology, Jinling Hospital, Nanjing Clinical Medical College of Second Military Medical University, Nanjing, 'bepartment of Medical Oncology, Jinling Hospital, Nanjing Clinical School of Southern Medical University, Nanjing, 'Department of Medical Oncology, Jinling Hospital, School of Medicine, Nanjing University, Nanjing, PR China
\end{abstract}

\section{Key Words}

MicroRNA-149 • FOXM1 • Chemoresistance • 5-Fluorouracil • Colorectal cancer

\begin{abstract}
Background/Aims: Previously, we have shown that microRNA (miR)-149 suppresses the migration and invasion of colorectal cancer (CRC) cells by targeting forkhead box transcription factor (FOXM1). However, the roles of miR-149 in the chemoresistance of CRC cells to 5 -Fluorouracil (5-FU) is unclear. The aim of this study is to investigate whether miR-149 targets FOXM1 to regulate the 5-FU resistance of CRC. Methods: The qRT-PCR assay was performed to detect the expression of miR-149 in 5-FU-resistant CRC cells (HCT-8/5-FU and LoVo/5FU) and their parental CRC cells (HCT-8 and LoVo). Also, the effects of miR-149 expression on the sensitivity of CRC cells to 5-FU were determined by gain- and loss-of-function assays. Finally, whether miR-149 regulates the 5-FU resistance of CRC cells by targeting the mammalian Forkhead Box M1 (FOXM1) was investigated. Results: The expression of miR-149 was significantly downregulated in 5-FU-resistant CRC cells in comparison with their parental CRC cells. Re-expression of miR-149 could enhance the 5-FU sensitivity of 5-FU-resistant CRC cells by increasing 5 -FU-inducing apoptosis, while downregulation of miR-149 could decrease the 5-FU sensitivity of parental CRC cells by decreasing 5-FU-inducing apoptosis. In addition, the luciferase assay indicated that miR-149 could bind to the $3^{\prime}$-UTR sequence of FOXM1 mRNA. The silencing of FOXM1 could mimic the effect of miR-149 upregulation on the 5-FU resistance of 5-FU-resistant CRC cells. Furthermore, the expression of miR-149 in the 5-FUresponding $C R C$ tissues was significantly higher than that in the non-responding tissues and inversely correlated with FOXM1 mRNA level. Conclusions: MiR-149 reverses the resistance of CRC cells to 5-FU by directly targeting FOXM1. Thus, targeting miR-149/FOXM1 signaling will be a potential strategy in the treatment of 5-FU-chemoresistant CRC.




\section{Introduction}

Colorectal cancer (CRC) is the third leading cause of cancer-related deaths around the world [1]. Chemotherapeutic agents serve as important adjuvant therapies for CRC treatment. 5-Fluorouracil (5-FU) is one of the chemotherapeutic drugs most widely used alone or combined with other drugs for patients with advanced CRC [2]. Adjuvant 5-FU treatment has yielded a good survival rate, but the failure of treatment in over $90 \%$ of CRC patients resulted from therapeutic resistance [3]. Currently, the mechanisms of 5-FU resistance in CRC remains poorly understood. Thus, elucidation of molecular mechanisms involved in chemoresistance will be helpful to develop novel strategies for the treatment of patients with 5-FU-resistant CRC.

MicroRNAs (miRNAs) are a class of 17 25 nucleotides small non-coding RNAs, which regulate gene expression primarily by binding to 3'-untranslated regions (3'-UTR) of specific target mRNAs to suppress translation and occasionally also induce their degradation [4]. Increasing evidence has shown that dysregulation of miRNAs play critical roles in various physiological and pathological processes including growth, differentiation, apoptosis and chemo- or radioresistance [5-8]. Meanwhile, dysregulated miRNAs is also found to be correlated with CRC progression [9, 10]. Previously, we have reported that reduced miR-149 was significantly correlated with lymph node metastasis, distant metastasis and TNM stage of CRC patients, and that status of miR-149 expression may be an independent prognostic factor [11]. Importantly, it was also observed that re-expression of miR-149 could inhibit migration and invasion of CRC cells via downregulation of MMPs, VEGF-A and UPAR, at least partially by targeting the transcription factor FOXM1. However, the correlation of dysregulated miR-149 with chemoresistance of CRC cells is unclear and there are no such reports. Here, we investigated whether miR-149 plays a role in the resistance of CRC cells to 5-FU and further explore the possible molecular mechanisms using 5-FU-resistant CRC cell models.

In the present study, we showed that re-expression of miR-149 could reverse the 5-FU resistance of CRC cells via enhancing 5-FU-induced apoptosis, at partially by targeting FOXM1. Our data indicated a novel regulatory pathway for 5-FU resistance involving miR149 and possibly provide valuable insight into CRC therapy in future. Thus, target miR-149/ FOXM1 signaling will be a potential strategy for the treatment of 5-FU-chemoresistant CRC patients.

\section{Materials and Methods}

\section{Cell culture}

Human CRC cell lines (HCT-8 and LoVo) were cultured at $37^{\circ} \mathrm{C}$ in RPMI- 1640 medium supplemented with $10 \%$ calf serum, $100 \mathrm{U} / \mathrm{mL}$ penicillin, and $100 \mu \mathrm{g} / \mathrm{mL}$ streptomycin, in an atmosphere of $5 \% \mathrm{CO}_{2}$ at saturation humidity. Two human 5-FU-resistant CRC cell lines (HCT-8/5-FU and LoVo/5-FU) was established and cultured in RMPI 1640 supplemented with $15.0 \mu \mathrm{g} / \mathrm{ml}$ and $6.5 \mu \mathrm{g} / \mathrm{ml} \mathrm{5-FU}$, respectively. Cells in logarithmic growth were used in all experiments.

\section{Taqman quantitative reverse transcription ( $q R T)$-PCR assay}

The expression levels of miR-149 and FOXM1 mRNA were quantified by using miRNA-specific TaqMan MiRNA Assay Kit (Applied Biosystems) and the SYBR Green reporter following manufacturer's protocol as described previously [9].

\section{Western blotting assay}

The cells were lysed using a modified radioimmuno precipitation assay buffer $(50 \mathrm{mM}$ Tris- $\mathrm{HCl}(\mathrm{pH}$ 7.4), $1 \%$ NP-40, $0.25 \%$ sodium deoxycholate, $150 \mathrm{mM} \mathrm{NaCl}, 1 \mathrm{mM}$ ethylenediaminetetraacetic acid, protease inhibitor cocktail complete. Amounts of total protein extracts were determined using BCA assay and samples were stored at $-134^{\circ} \mathrm{C}$ until use. Proteins were separated by sodium dodecyl sulfate-polyacrylamide 


\section{Cellular Physiology Cell Physiol Biochem 2016;39:617-629 \begin{tabular}{ll|l} 
DOI: 10.1159/000445653 & $\begin{array}{l}\text { O 2016 The Author(s). Published by S. Karger AG, Basel } \\
\text { www.karger.com/cpb }\end{array}$
\end{tabular}}

Liu et al.: MicroRNA-149 and 5-FU Resistance in Colorectal Cancer

gel electrophoresis (SDS-PAGE). All the specific antibodies against phosphorylated AKT (pAKT) (Ser473), total AKT, FOXM1, total PARP, cleaved PARP or GAPDH were purchased from Santa Cruz Biotechnology (Santa Cruz, CA, USA). The membranes were then incubated with a horseradish peroxidase-conjugated secondary antibody (Sigma, USA). Probing and detection of target proteins was performed by an enhanced chemiluminescence (ECL) assay after antibody binding, and light emission was captured on Kodak X-ray films.

In vitro chemotherapy assay

Single-cell suspensions were prepared and dispersed in 96-well plates. After incubation for $72 \mathrm{~h}$ with the 5-FU compounds (Sigma, Saint Louis, MO, USA), the $0.5 \mathrm{mg} / \mathrm{mL}$ of MTT solution (Sigma, USA) was added. Following incubation for $4 \mathrm{~h}, 100 \mu \mathrm{L}$ of extraction buffer were added to each well. After an overnight incubation, absorbance at $490 \mathrm{~nm}$ was measured using a microplate reader.

Transfection of miRNA mimics, inhibitors or plasmid vectors

MiR-149/mimics or inhibitor (anti-miR-149) and their negative control oligonucleotides (miR-NC/ mimics or anti-miR-NC) were obtained from Ambion Inc (Austin, TX, USA). The small hairpin RNA (shRNA) plasmid vector targeting FOXM1 (pSil/shFOXM1) and control vector (pSil/shcontrol) were successfully constructed previously [12]. The recombinant vectors were confirmed by the digestion analysis of restriction endonuclease and DNA sequencing. Transient or stable transfections were performed using Lipofectamine $^{\mathrm{TM}} 2000$ (Invitrogen, USA).

Luciferase reporter assay

To construct a luciferase reporter vector, the FOXM1-3'-UTR fragment containing putative binding sites for miR-149 (3583-3605bp) was amplified by PCR and subcloned into the downstream of luciferase gene in the pLUC luciferase vector (Ambion, USA) and named pLUC/FOXM1-3'-UTR-wt. Site-directed mutagenesis of the miR-149 target-site in the pLUC/FOXM1-3'-UTR-wt was performed using the QuickChange Site-Directed Mutagenesis Kit (Stratagene, Germany) and named pLUC/FOXM1-3'-UTR-mut. For luciferase reporter assays, HCT-8/5-FU or LoVo/5-FU cells $\left(3 \times 10^{5}\right)$ were plated in a 24-well plate and then cotransfected with $100 \mathrm{ng}$ of pLUC/FOXM1-3'-UTR-wt or pLUC/FOXM1-3'-UTR-wt and $50 \mathrm{nM}$ of miR-149/mimics (or miR-NC/mimics) or anti-miR-149 (or anti-miR-NC) using Lipofectamine 2000. Fortyeight hours after transfection, cells were harvested and assayed with Dual-Luciferase Reporter Assay kit (Promega, USA) according to the manufacturer's instructions.

Patients and tissue samples

A total of 24 CRC tissues were collected from patients with advanced CRC who received chemotherapy at Department of Medical Oncology, Jinling Hospital between May 2014 and July 2015. Patients met all of the following criteria: a histological diagnosis of CRC with at least one measurable lesion; a clinical stage of IV; received at least 4 cycles of 5-FU-based chemotherapy every 3 weeks including modified FOLFOX-6 (oxaliplatin $85 \mathrm{mg} / \mathrm{m}^{2}$, leucovorin $400 \mathrm{mg} / \mathrm{m}^{2}$, a bolus of $5-\mathrm{FU} 400 \mathrm{mg} / \mathrm{m}^{2}$ followed by a $46-\mathrm{hr}$ infusion of $2400 \mathrm{mg} / \mathrm{m}^{2}$ ), XELOX (Xeloda $1250 \mathrm{mg} / \mathrm{m} 2$ orally on days 1-14, oxaliplatin $130 \mathrm{mg} / \mathrm{m} 2$ on day 1 ) or FOLFIRI (irinotecan $180 \mathrm{mg} / \mathrm{m} 2$, leucovorin $200 \mathrm{mg} / \mathrm{m} 2$, a bolus of $5-\mathrm{FU} 400 \mathrm{mg} / \mathrm{m}^{2}$ followed by a $46-\mathrm{hr}$ infusion of $2400 \mathrm{mg} / \mathrm{m}^{2}$ ). Tumor response was examined by computed tomography and evaluated according to the 1.1 version of Response Evaluation Criteria in Solid Tumors (RECIST 1.1) as complete response (CR), partial response (PR), stable disease (SD), or progressive disease (PD). The chemotherapy-responding subjects were defined as the total of those achieved CR, PR or SD responses. Written permission to use human tumor tissues was obtained from the patients.

\section{Statistical analysis}

Data are expressed as mean \pm standard deviation (SD). The difference between miR-149 and FOXM1 mRNA expressions in chemotherapy-responding and non-responding CRC tissues was evaluated using Student's $t$-test. Statistical analysis was performed using SPSS 17.0 software program (SPSS Inc., Chicago, Illinois, USA). 


\section{Results}

Expression of miR-149 is significantly downregulated in 5-FU-resistant CRC cells

To understand the molecular mechanisms involved in the 5-FU resistance of CRC, 5-FUresistant human CRC cell lines (HCT-8/5-FU or LoVo/5-FU) from parental HCT-8 or LoVo cell line were established in our lab after the selection by drug pressure. In vitro chemosensitivity assays indicated that HCT-8/5-FU ( $\left.\mathrm{IC}_{50}: 34.8 \mu \mathrm{g} / \mathrm{ml}\right)$ and LoVo/5-FU ( $\left.\mathrm{IC}_{50}: 73.7 \mu \mathrm{g} / \mathrm{ml}\right)$ cells were more resistant against 5-FU compared to the parental HCT-8 $\left(\mathrm{IC}_{50}: 2.8 \mu \mathrm{g} / \mathrm{ml}\right)$ and LoVo $\left(\mathrm{IC}_{50}: 11.8 \mu \mathrm{g} / \mathrm{ml}\right)$ cells, with about 13.6- and 6.3-fold increase in $\mathrm{IC}_{50}$ values, respectively (Fig. 1A). To investigate the correlation of miR-149 expression with 5-FU resistance of CRC, qRT-PCR was performed to detect the expression of miR-149 in both 5-FU-resistant and parental CRC cells. It was observed that the expression of miR-149 was significantly downregulated in HCT-8/5-FU and LoVo/5-FU cells, compared to that in parental cells (Fig. 1B). Activation of AKT has been reported to play a role in tumor chemoresistance. Here, we also detected the protein expression of pAKT and total AKT in 5-FU-resistant and parental CRC cells by Western blot. It was observed that the expression levels of both pAKT and total AKT proteins were significantly higher in HCT-8/5-FU and LoVO/5-FU than parental HCT-8 and LoVo cells (Fig. 1C). Thus, reduced miR-149 levels and activation of AKT could account for 5-FU resistance of CRC cells.

Fig. 1. Expression of miR-149 in 5-FU-resistant and parental CRC cells. (A) MTT assay was conducted to detect the growth of 5-FU-resistant CRC cells (HCT-8/5FU and LoVo/5-FU) and their parental CRC cells (HCT-8 and LoVo) at different concentrations of 5-FU, respectively. The $\mathrm{IC}_{50}$ values of 5-FU to 5-FU-resistant and parental CRC cells were shown. (B) qRT-PCR detection of miR149 expression in 5-FU-resistant CRC cells (HCT-8/5-FU and LoVo/5-FU) and their parental CRC cells (HCT-8 and LoVo). U6 was used

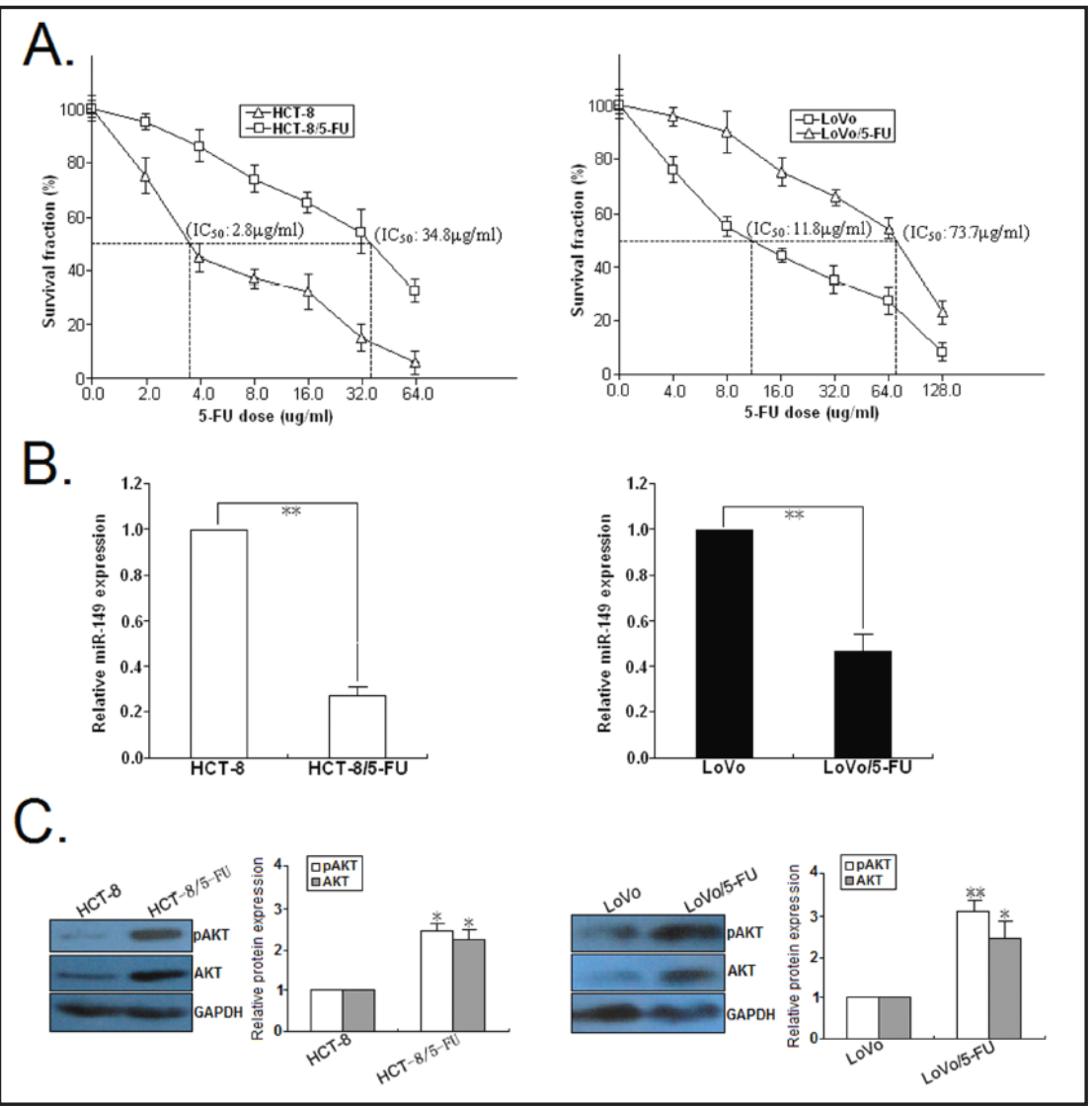

as an internal control. (C) Western blot detect the expression of pAKT and total AKT proteins in 5-FU-resistant CRC cells (HCT-8/5-FU and LoVo/5-FU) and their parental CRC cells (HCT-8 and LoVo). GAPDH was used as an internal control. Results represent the average of three independent experiments (mean \pm SD). ** $P<0.01$. 
A. B

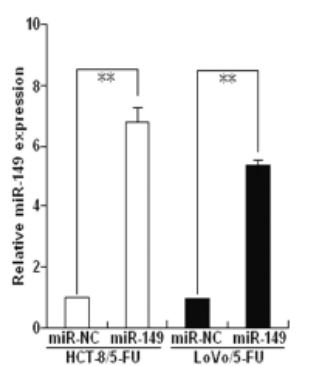

B.

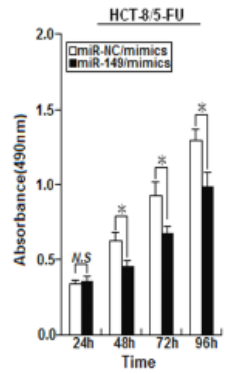

D.
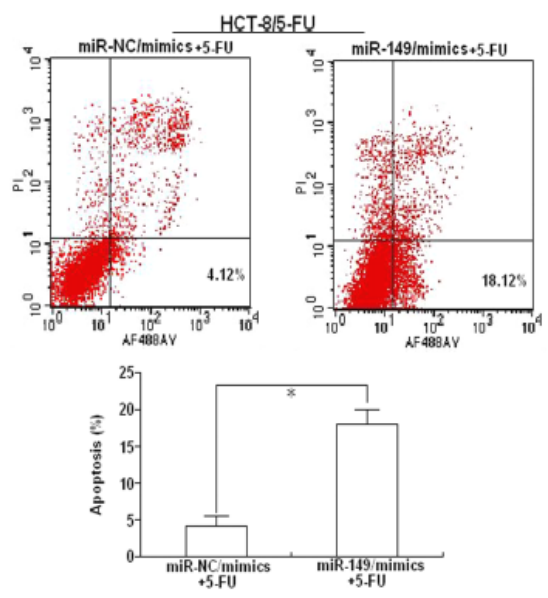

E.

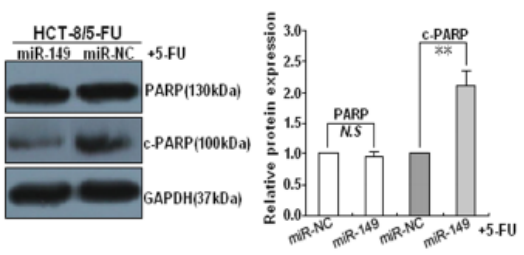

C.

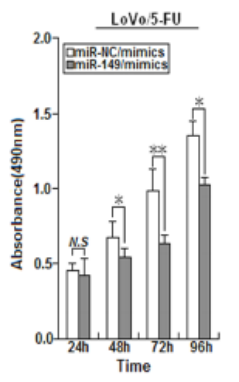

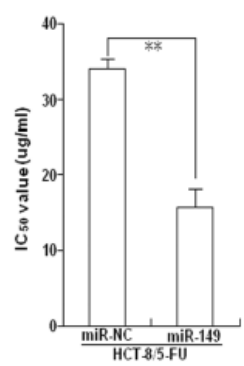

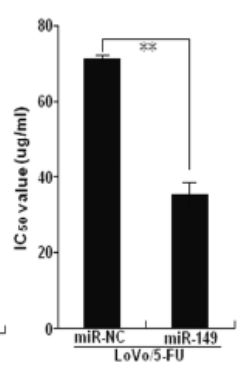

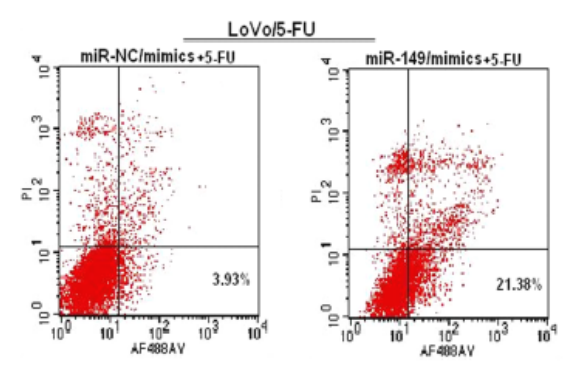
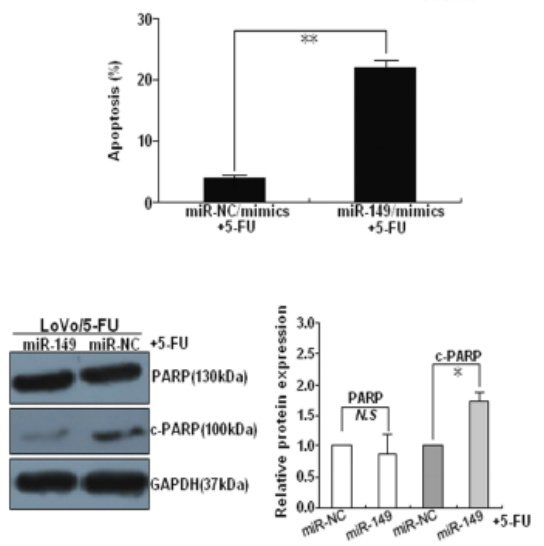

Fig. 2. Effects of miR-149 upregulation on the sensitivity of 5-FU-resistant CRC cells to 5-FU. (A) qRT-PCR detection of miR-149 expression in HCT-8/5-FU and LoVo/5-FU cells transfected with miR-149/mimics or miR-NC/mimics, respectively. U6 was used as an internal control. (B) MTT analysis of growth in miR-NC/ mimics or miR-149/mimics-transfected HCT-8/5-FU or LoVo/5-FU cells at different time point $(24 \mathrm{~h}, 48 \mathrm{~h}$, $72 \mathrm{~h}$ or $96 \mathrm{~h}$ ). (C) MMT assay was conducted to detect the $\mathrm{IC}_{50}$ values of 5-FU to HCT-8/5-FU and LoVo/5-FU cells transfected with miR-149/mimics or miR-NC/mimics, respectively. (D) Flow cytometric detection of apoptosis in miR-149/mimics or miR-NC/mimics-transfected HCT-8/5-FU and LoVo/5-FU cells combined with 5-FU treatment (HCT-8/5-FU: $15.0 \mu \mathrm{g} / \mathrm{ml}$; LoVo/5-FU: $6.5 \mu \mathrm{g} / \mathrm{ml}$ ). (E) Western blotting detection of PARP or c-PARP protein expression in miR-149/mimics or miR-NC/mimics-transfected HCT-8/5-FU and LoVo/5-FU cells combined with 5-FU treatment (HCT-8/5-FU: $15.0 \mu \mathrm{g} / \mathrm{ml}$; LoVo/5-FU: 6.5 g/ml). GAPDH was used as an internal control. Results represent the average of three independent experiments (mean \pm SD). ${ }^{*} P<0.05,{ }^{* *} P<0.01$. N.S: not significance versus control.

Upregulation of miR-149 significantly increases the sensitivity of 5-FU-resistant CRC cells to 5 - FU

To investigate the effects of miR-149 expression on the 5-FU sensitivity of CRC cells, HCT-8/5-FU and LoVo/5-FU cells were transiently transfected with miR-149/mimics or miR-NC/mimics, respectively. The upregulation of miR-149 was confirmed in miR-149/ 
A.

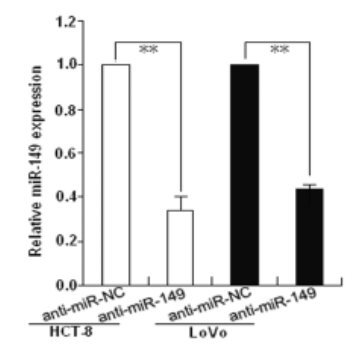

C.
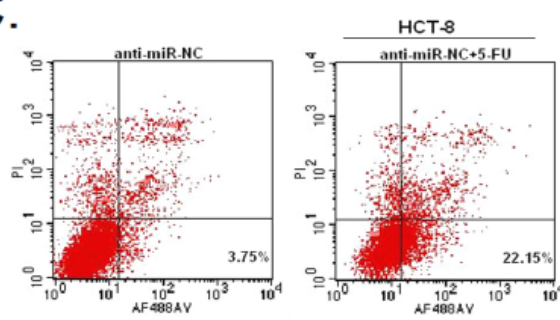

LoVo
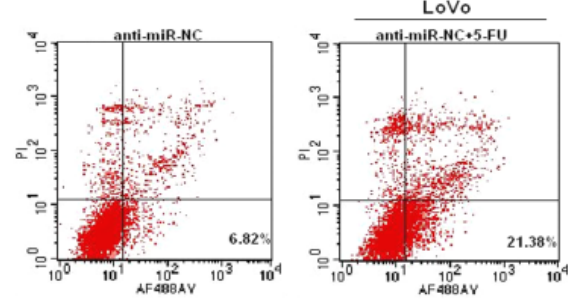
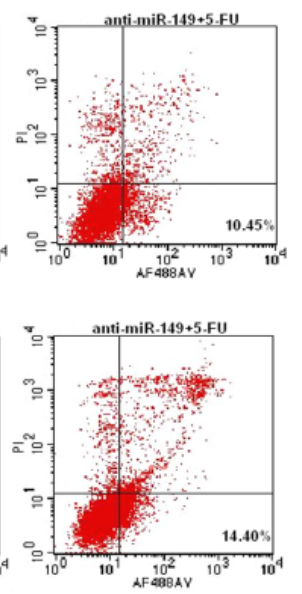

B.
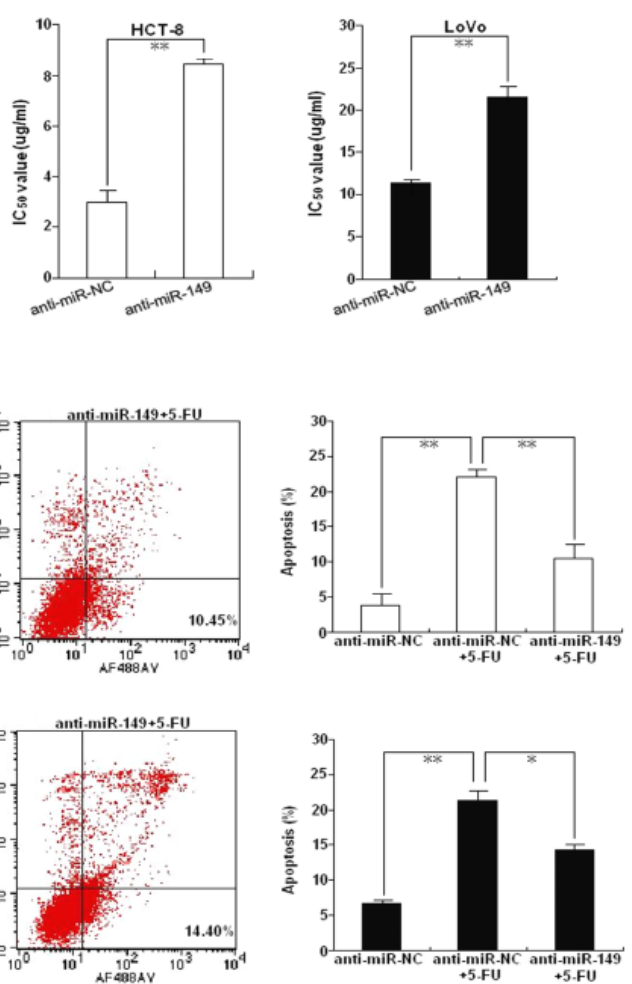

D.
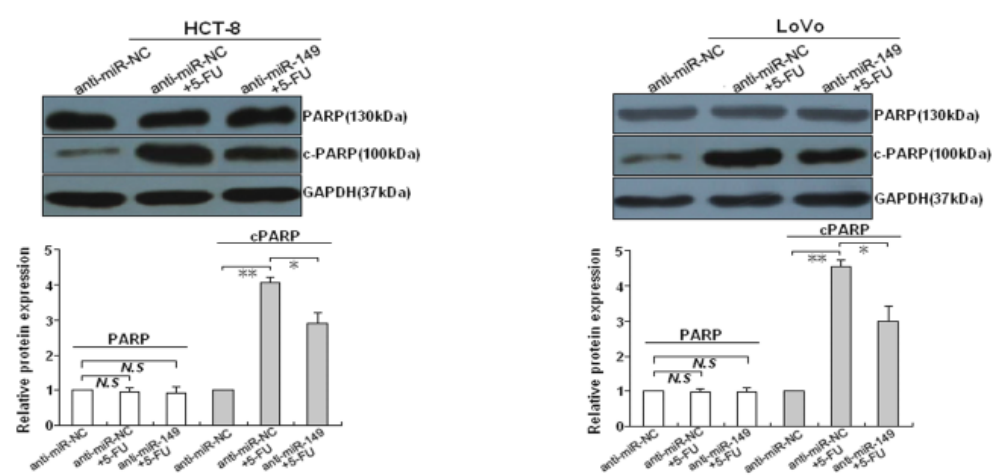

Fig. 3. Effects of miR-149 downregulation on the sensitivity of parental CRC cells to 5-FU. (A) qRT-PCR detection of miR-149 expression in HCT-8 and LoVo cells transfected with anti-miR-149 or anti-miR-NC, respectively. U6 was used as an internal control. (B) MTT assay was conducted to detect the $\mathrm{IC}_{50}$ values of 5-FU to HCT-8 and LoVo cells transfected with anti-miR-149 or anti-miR-NC, respectively. (C) Flow cytometric detection of apoptosis in anti-miR-149 or anti-miR-NC -transfected HCT-8 and LoVo cells combined with 5-FU treatment (HCT-8: $1.5 \mu \mathrm{g} / \mathrm{ml}$; LoVo: $3.0 \mu \mathrm{g} / \mathrm{ml}$ ). (D) Western blotting detection of PARP or c-PARP protein expression in anti-miR-149 or anti-miR-NC -transfected HCT-8 and LoVo cells combined with 5-FU treatment (HCT-8: $1.5 \mu \mathrm{g} / \mathrm{ml}$; LoVo: $3.0 \mu \mathrm{g} / \mathrm{ml}$ )). GAPDH was used as an internal control. Results represent the average of three independent experiments (mean \pm SD). ${ }^{*} P<0.05$, ${ }^{* *} P<0.01$. N.S: not significance versus control.

mimics-transfected cells by qRT-PCR (Fig. 2A). Then, we analyzed the effect of miR-149 upregulation on growth of 5-FU-resistant CRC cells by MTT assay, and results indicated that upegulation of miR-149 moderately inhibit growth of HCT-8/5-FU or LoVo/5-FU cells (Fig. 2B). Next, the effect of miR-149 upregulation on the $\mathrm{IC}_{50}$ values of 5-FU in chemoresistant 


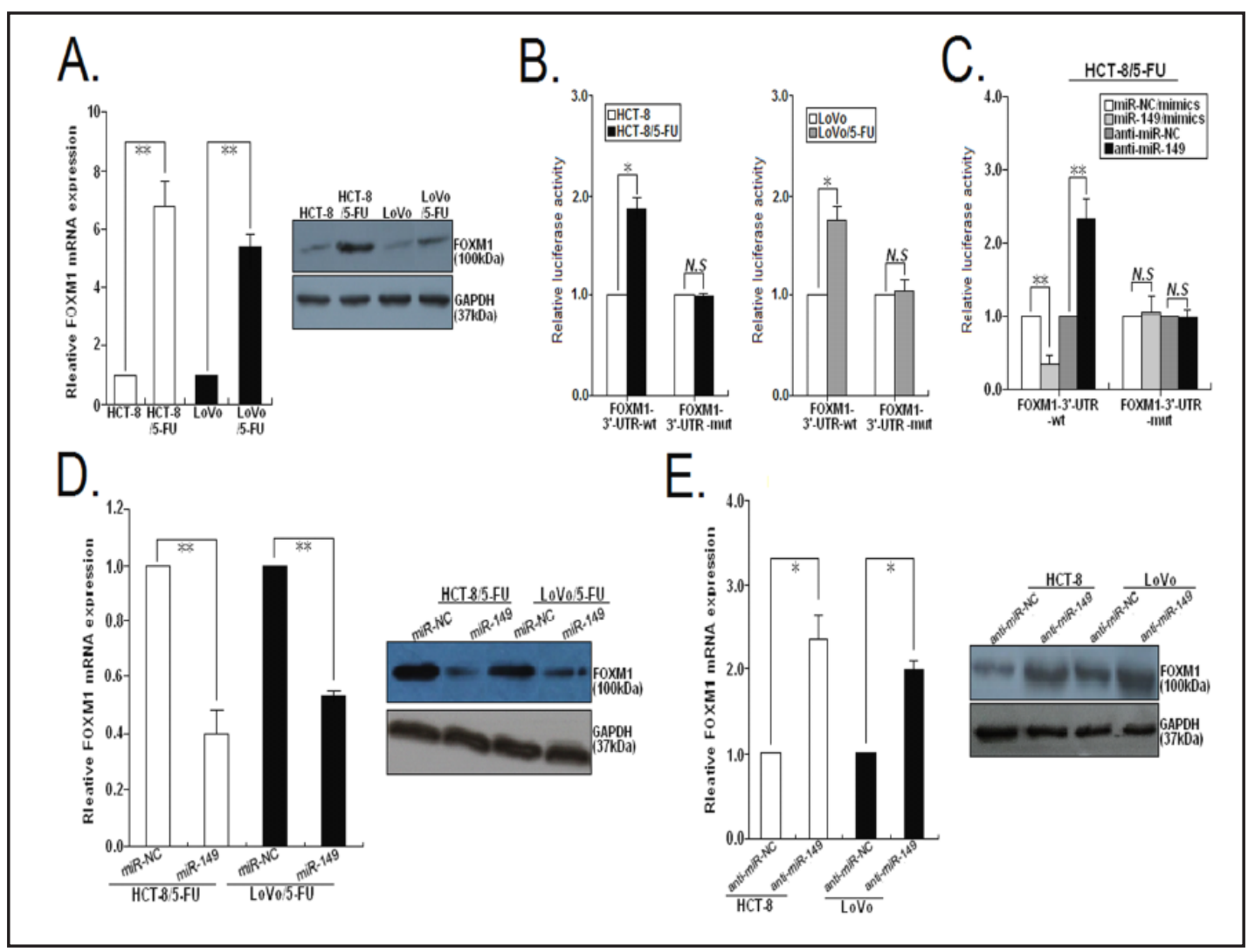

Fig. 4. FOXM1 is identified as a direct target of miR-149. (A) qRT-PCR and Western blotting detection of FOXM1 mRNA and protein expression in 5-FU-resistant CRC cells (HCT-8/5-FU and LoVo/5-FU) and their parental CRC cells (HCT-8 and LoVo), respectively. (B) Relative luciferase activity was analyzed. pLUC/FOXM13'-UTR-wt or pLUC/FOXM1-3'-UTR-mut vector was transfected into HCT-8/5-FU (or HCT-8) or LoVo/5-FU (LoVo) cells. Cells lysates were prepared after $48 \mathrm{~h}$ for measuring luciferase activity, which was normalized to normalized to Renilla luciferase activity. (C) Relative luciferase activity was analyzed. pLUC/FOXM1-3'UTR-wt or pLUC/FOXM1-3'-UTR-mut vector and miR-149/mimics (or miR-NC/mimics) or anti-miR-149 (or anti-miR-NC) were co-transfected into HCT-8/5-FU or LoVo/5-FU cells. Cells lysates were prepared after $48 \mathrm{~h}$ for measuring luciferase activity, which was normalized to normalized to Renilla luciferase activity. (D) qRT-PCR and Western blotting detection of FOXM1 mRNA and protein expression in miR-149/mimics or miR-NC/mimics-transfected HCT-8/5-FU and LoVo/5-FU cells, respectively. (E) qRT-PCR and Western blotting detection of FOXM1 mRNA and protein expression in anti-miR-149 or anti-miR-NC-transfected HCT-8 and LoVo cells, respectively. GAPDH was used as an internal control. Results represent the average of three independent experiments (mean $\pm \mathrm{SD}$ ). ${ }^{*} P<0.05$, ${ }^{* *} P<0.01$. N.S: not significance versus control.

CRC cells was further determined (Fig. 2C). It was observed that transfection of miR-149/ mimics could induce a remarkable reversal of 5-FU resistance in HCT-8/5-FU cells, with the 5 -Fu IC ${ }_{50}$ decreased from $34.8 \mu \mathrm{g} / \mathrm{ml}$ to $15.5 \mu \mathrm{g} / \mathrm{ml}$ in comparison with the miR-NC/ mimics-transfected cells. Likewise, miR-149/mimics induced a remarkable reversal of 5-FU resistance in LoVo/5-FU cells, with the 5 - $\mathrm{Fu} \mathrm{IC}_{50}$ decreased from $73.7 \mu \mathrm{g} / \mathrm{ml}$ to $35.8 \mu \mathrm{g} /$ $\mathrm{ml}$ in comparison with the miR-NC/mimics-transfected cells. Furthermore, we analyzed the effects of miR-149 upregulation on apoptosis of 5-FU-resistant CRC cells. As shown in Fig. 2D, upregulation of miR-149 could significantly increase apoptosis of HCT-8/5-FU and LoVo/5-FU cells in response to $15.0 \mu \mathrm{g} / \mathrm{ml}$ and $6.5 \mu \mathrm{g} / \mathrm{ml}$ of 5-FU, respectively. Also, induction of PARP cleavage by 5-FU was significantly increased in miR-149-expressing cells as compared with the control cells (Fig. 2E). Therefore, upregulation of miR-149 increased the 5-FU sensitivity of CRC cells resulting in activation of apoptosis. 
Downregulation of miR-149 significantly reduces the sensitivity of 5-FU-sensitive CRC cells to 5 - FU

To further investigate the effects of miR-149 downregulation on the sensitivity of CRC cells to 5-FU, parental HCT-8 and LoVo cells were transiently transfected with anti-miR-149 or anti-miR-NC, respectively. The downregulation of miR-149 was confirmed in miR-149/ inhibitor-transfected cells by qRT-PCR, compared to the control cells (Fig. 3A). Anti-miR-149 could decrease the 5-FU sensitivity in parental HCT-8 cells, with the $\mathrm{IC}_{50}$ increased from $2.8 \mu \mathrm{g} / \mathrm{ml}$ to $8.45 \mu \mathrm{g} / \mathrm{ml}$ in comparison with the anti-miR-NC-transfected cells (Fig. 3B). Likewise, transfection of anti-miR-149 could decrease the 5-FU sensitivity in parental LoVo cells, with the $\mathrm{IC}_{50}$ increased from $11.8 \mu \mathrm{g} / \mathrm{ml}$ to $21.6 \mu \mathrm{g} / \mathrm{ml}$ in comparison with the antimiR-NC-transfected cells. Downregulation of miR-149 could significantly decrease apoptosis of HCT-8 and LoVo cells in response to $1.5 \mu \mathrm{g} / \mathrm{ml}$ and $3.0 \mu \mathrm{g} / \mathrm{ml}$ of 5 -FU, respectively (Fig. 3C). Also, induction of PARP cleavage by 5-FU was significantly decreased in miR-149downregulating cells as compared with the control cells (Fig. 3D). Thus, downregulation of miR-149 decreases the 5-FU sensitivity of 5-FU-sensitive CRC cells by reducing 5-FU-induced apoptosis.

FOXM1 is identified as a target of miR-149 in 5-FU-resistant CRC cells

Previously, we have shown that miR-149 suppresses CRC migration and invasion by directly targeting FOXM1. However, whether miR-149 regulates the sensitivity of CRC cells to 5-FU by targeting FOXM1 is still unclear. First, we detected the expressions of FOXM1 mRNA and protein in HCT-8/5-FU, LoVo/5-FU and their parental cells, and found out that both the FOXM1 mRNA and protein levels in 5-FU-resistant CRC cells were significantly higher than those in parental cells (Fig. 4A), suggesting that the expression of FOXM1 inversely correlated with the miR-149 level in CRC cells.

The 3'-untranslated region (UTR) of FOXM1 gene stood out because of the presence of one evolutionarily conserved binding site (3583 3605bp) for miR-149, and the 3'-UTR sequence of FOXM1 (3'-UTR-wt) or the mutant sequence (3'-UTR-mut) was previously cloned into the luciferase reporter vector pLUC, which was named pLUC/FOXM1-3'-UTR-wt and pLUC/FOXM1-3'-UTR-mut, respectively. Then, those two vectors were transfected into 5-FU-resistant CRC cells (HCT-8/5-FU and LoVo/5-FU) and their parental CRC cells (HCT-8 and LoVo), respectively. $48 \mathrm{~h}$ after transfection, the luciferase activity was determined. It was observed that the luciferase activity of pLUC/FOXM1-3'-UTR-wt was significantly increased in HCT-8/5-FU $(P<0.05)$ or LoVo/5-FU $(P<0.05)$ cells, compared to parental HCT-8 or LoVo cells (Fig. 4B), indirectly suggesting that the relative expression of miR-149 in 5-FU-resistant CRC cells was higher than parental CRC cells. Next, those two luciferase reporter vectors and miR-149/mimics (or miR-NC/mimics) or anti-miR-149 (or anti-miR-NC) were cotransfected into HCT-8/5-FU cells, and 48h after co-transfection, the luciferase activity was determined (Fig. 4C). Results showed that the luciferase activity of pLUC/FOXM1-3'-UTRwt was significantly reduced by miR-149/mimics, compared to miR-NC/mimics $(P<0.01)$, and increased by anti-miR-149, compared to anti-miR-NC $(P<0.01)$. However, the luciferase activity of pLUC/FOXM1-3'-UTR-mut was not changed by the simultaneous co-transfection with miR-149/mimics or anti-miR-149 $(P>0.05)$. Next, we analyzed the effect of miR-149 expression on the levels of FOXM1 mRNA and protein. Transfection of miR-149/mimics decreased both the expressions of FOXM1 mRNA and protein in HCT-8/5-FU and LoVo/5FU cells (Fig. 4D), while transfection of anti-miR-149 induced the increased expression of FOXM1mRNA and protein in parental HCT-8 and LoVo cells (Fig. 4E). These results indicate that FOXM1 is a direct target of miR-149 in 5-FU-resistant CRC cells.

Silencing of FOXM1 mimics the effect of miR-149 upregulation on 5-FU sensitivity in 5-FUresistant CRC cells

Further, we investigated whether FOXM1 is a functional target of miR-149 in 5-FUresistantCRC cells. The previously constructed small interfering RNA (siRNA) vector targeting FOXM1 (pSil/shFOXM1) and control vector (pSil/shcontrol) were stably transfected into 


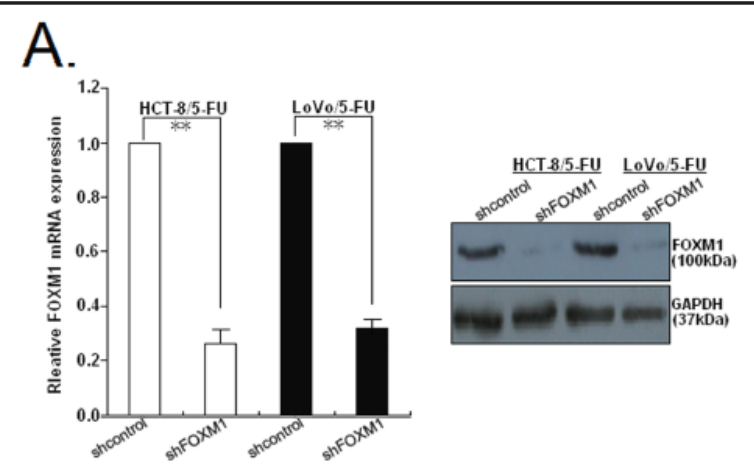

B.

C.
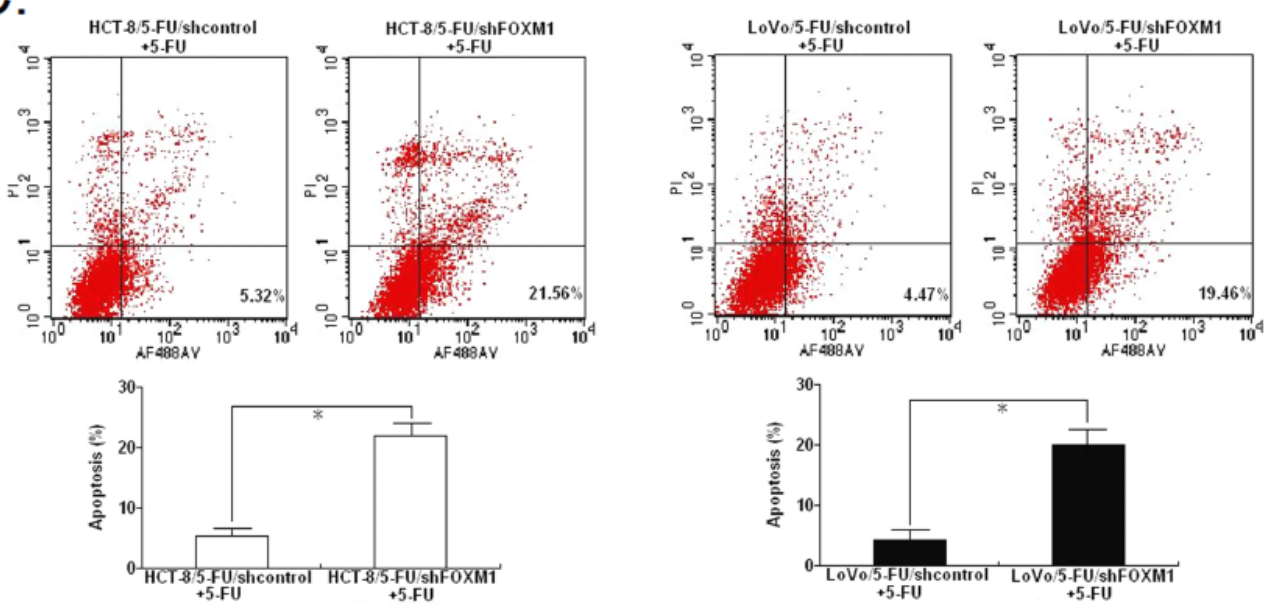

D.
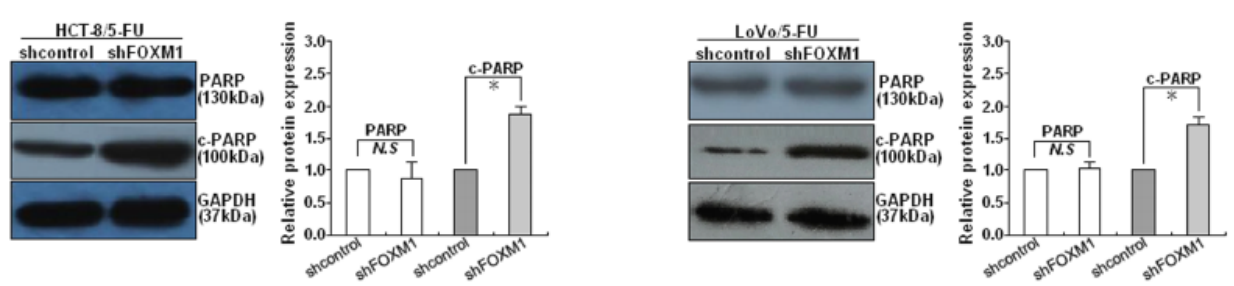

Fig. 5. Effects of FOXM1 downregulation on the sensitivity of 5-FU-resistant CRC cells to 5-FU. (A) qRTPCR and Western blotting detection of FOXM1 mRNA and protein expression in HCT-8/5-FU/shFOXM1 and LoVo/5-FU/shFOXM1 cells or their control cells (HCT-8/5-FU/shcontrol and LoVo/5-FU/shcontrol). GAPDH was used as an internal control. (B) MTT assay was conducted to detect the $\mathrm{IC}_{50}$ values of 5-FU to HCT8/5-FU/shFOXM1 and LoVo/5-FU/shFOXM1 cells or their control cells. (C) Flow cytometric detection of apoptosis in miR-149/mimics HCT-8/5-FU/shFOXM1 and LoVo/5-FU/shFOXM1 cells or their control cells combined with 5-FU treatment (HCT-8/5-FU: $15.0 \mu \mathrm{g} / \mathrm{ml}$; LoVo/5-FU: $6.5 \mu \mathrm{g} / \mathrm{ml}$ ). (D) Western blotting detection of PARP or c-PARP protein expression in HCT-8/5-FU/shFOXM1 and LoVo/5-FU/shFOXM1 cells or their control cells combined with 5-FU treatment (HCT-8/5-FU: $15.0 \mu \mathrm{g} / \mathrm{ml}$; LoVo/5-FU: $6.5 \mu \mathrm{g} / \mathrm{ml}$ ). GAPDH was used as an internal control. Results represent the average of three independent experiments (mean \pm SD). ${ }^{*} P<0.05,{ }^{* *} P<0.01$. N.S: not significance versus control.

HCT-8/5-FU and LoVo/5-FU cells, which were named HCT-8/5-FU/shFOXM1 (or HCT-8/5FU/shcontrol) and LoVo/5-FU/shFOXM1 (or LoVo/5-FU/shcontrol), respectively. Then, qRT-PCR and Western Blotting confirmed the knockdown of FOXM1 in HCT-8/5-FU and LoVo/5-FU cells (Fig. 5A). MTT assay was performed to investigate the effects of FOXM1 downregulation on the 5-Fu IC ${ }_{50}$ of 5-FU-resistant CRC cells. As shown in Fig. 5B, knockdown 


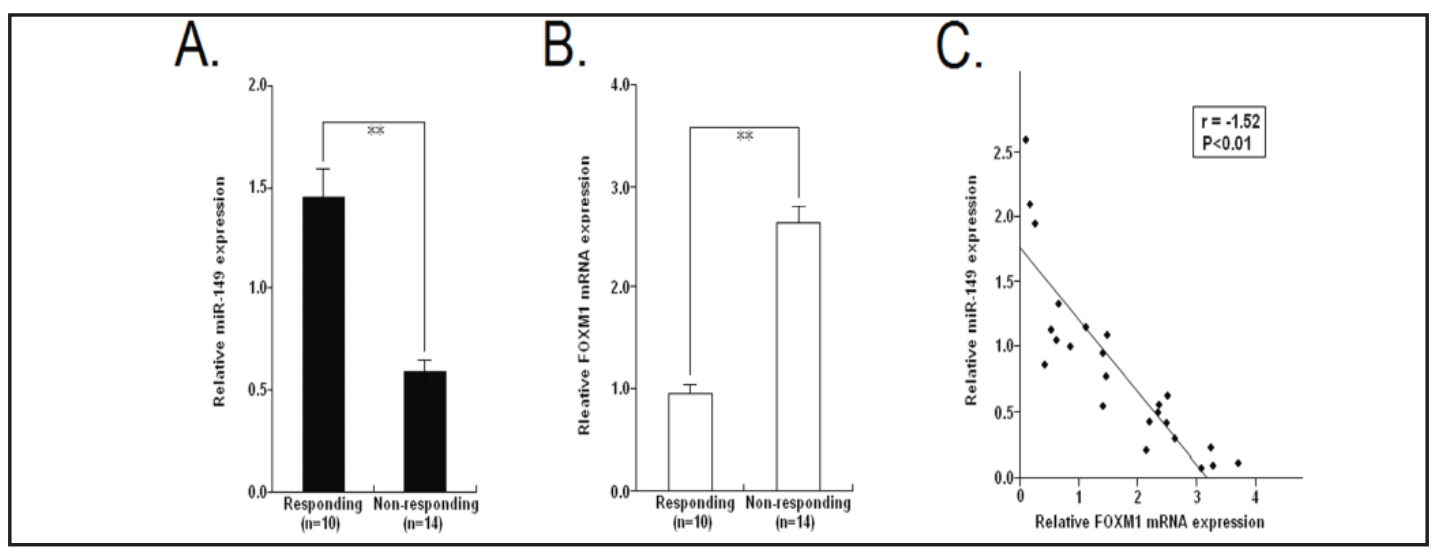

Fig. 6. Expression of miR-149 inversely correlates with FOXM1 mRNA expression in CRC tissues. (A) qRTPCR detection of miR-149 expression in the responding CRC tissues $(n=10)$ and non-responding CRC tissues $(n=14)$ from patients who received 5-FU-based chemotherapy. U6 was used as an internal control. (B) qRT-PCR detection of FOXM1 mRNA expression in the responding CRC tissues $(n=10)$ and non-responding CRC tissues $(\mathrm{n}=14$ ) from patients who received 5-FU-based chemotherapy. (C) A statistically significant inverse correlation between miR-149 and FOXM1 mRNA expression levels in CRC tissues ( $\mathrm{n}=24$; Spearman's correlation analysis, $\mathrm{r}=-1.52 ; P<0.01$ ). Results represent the average of three independent experiments (mean \pm SD). Corresponding $P$ values analyzed by Spearman correlation test are indicated. $* P<0.05$, $* * P<0.01$.

of FOXM1 induced a significant reversal of 5-FU resistance in HCT-5/5-FU and LoVo/5-FU cells, with the $5-\mathrm{Fu} \mathrm{IC}{ }_{50}$ decreased to $10.5 \mu \mathrm{g} / \mathrm{ml}(P<0.05)$ and $29.4 \mu \mathrm{g} / \mathrm{ml}(P<0.05)$ in comparison with the control cells. It was also observed that knockdown of FOXM1 increased the apoptosis in HCT-8/5-FU and LoVo/5-FU cells in response to the 5-FU treatment (Fig. 5C). Likewise, induction of PARP cleavage by 5 -FU was significantly increased in HCT-8/5FU/shFOXM1 and LoVo/5-FU/shFOXM1 cells, when compared to the control cells (Fig. 5D). These data suggest that knockdown of FOXM1 could mimic the effects of miR-149 upregulation on the 5-FU sensitivity of CRC cells.

Expression of miR-149 is downregulated in 5-FU-resistant CRC tissues and inversely correlates with FOXM1 expression

First, qRT-PCR was performed to detect the expression of miR-149 and FOXM1 mRNA in CRC tissues collected from patients who received 5-FU-based chemotherapy $(n=24)$. It was observed that the relative level of miR-149 in the chemotherapy-responding CRC tissues ( $\mathrm{n}=$ $10 ; 1.45 \pm 0.24)$ was significantly higher than that in the non-responding tissues $(\mathrm{n}=14 ; 0.53$ $\pm 0.15)(P<0.01$; Fig. 6A). Next, the expression of FOXM1 mRNA in CRC tissues was detected. In contrast to miR-149, the mean level of FOXM1 mRNA in the responding CRC tissues $(\mathrm{n}=$ $10 ; 1.84 \pm 0.12)$ was significantly lower than that in the non-responding tissues $(n=14 ; 2.64$ $\pm 0.25)(P<0.05$; Fig. 6B). In addition, the inverse correlation between miR-149 and FOXM1 mRNA levels was verified by linear regression analysis $(n=24, r=-1.52, P<0.01$; Fig. 6C).

\section{Discussion}

Chemoresistance is a major challenge to effective treatment in the patients with advanced CRC. It is urgent to develop novel strategies for overcoming the resistance to chemotherapeutic agents. In the present study, we showed that upregulation of miR-149 could reverse the 5-FU resistance of CRC cells via enhancing 5-FU-inducing apoptosis by targeting FOXM1. These data suggest that reduced miR-149 may be a major mechanism underlying the development of 5-FU resistance in CRC cells.

Hsa-miR-149, located on chromosome 2, has been proven to be an essential miRNA implicated in the development and progression of human malignancies. First, the 
polymorphism of miR-149 was found to be correlated with tumor susceptibility. An updated meta-analysis by Li et al. showed that the miR-149 rs2292832 T/C polymorphism may decrease the susceptibility of digestive cancers [13]. Wei and his colleagues reported that the miR-149 Rs2292832 polymorphism is involved in the susceptibility and local progression of papillary thyroid cancer in Chinese patients [14]. Huang et al. also showed that the CC genotype of miR-149 contributes to the progression and development, rather than the initiation of nasopharyngeal carcinoma [15]. Second, miR-149 is reported to function as a tumor suppressor in human cancers. Zhang et al. showed that miR-149 functions as a tumor suppressive miRNA and plays an important role in inhibiting the tumorigenesis of hepatocelluar carcinoma by modulating the AKT/mTOR pathway [16]. Wang et al. showed that miR-149 inhibits proliferation and cell cycle progression through the target of ZBTB2 in gastric cancer [17]. Pan et al. reported that miR-149 inhibits proliferation and invasion of glioma cells via blockade of AKT1 signaling [18]. Additionally, it was found out that miR149 suppresses breast cancer cell migration/invasion and metastasis by targeting GIT1 [19]. Likewise, our previous study indicated that miR-149 could suppress CRC migration and invasion [11]. Meanwhile, the correlations of miR-149 expression with tumor chemoresistance are currently reported. He and his colleagues have reported that methylation-regulated miR149 modulates chemoresistance by targeting GlcNAc N-deacetylase/N-sulfotransferase- 1 in breast cancer [20]. Also, it was shown that miR-128 and miR-149 could enhance the chemosensitivity of temozolomide by Rap1B-mediated cytoskeletal remodeling in glioblastoma [21]. However, the correlation of miR-149 expression with the resistance of $\mathrm{CRC}$ to $5-\mathrm{FU}$ is unclear and remains to be further elucidated.

Recently, the roles of miRNAs in the 5-FU chemoresistance of CRC are increasingly reported. Some miRNAs have been reported to enhance the 5-FU sensitivity of CRC cells. For instance, Li et al. showed that miR-218 functions synergistically with 5-FU to promote chemosensitivity by suppressing BIRC5 and TS in CRC [22]. He et al. showed that overexpression of miR-122 re-sensitizes 5-FU-resistant CRC cells to 5-FU through the inhibition of PKM2 in vitro and in vivo [23]. Similarly, other miRNAs are reported to induce the 5-FU resistance of CRC cells. For instance, Zhang et al. showed that miR-587 antagonizes 5-FU-induced apoptosis and confers drug resistance by regulating PPP2R1B expression in CRC [24]. Nishida et al. showed that miR-10b confers the resistance to 5-FU in CRC cells [25]. These data have implied the important roles of miRNAs in regulating the 5-FU sensitivity of CRC cells. To date, there are no reports about the correlation of miR-149 with the 5-FU sensitivity of CRC cells. In this study, we first detected the expression of miR-149 in two 5-FU-resistant and their parental CRC cells, and showed that the expression of mi-149 in 5-FU-resistant CRC cells was significantly higher than that in parental cells. Meanwhile, we also showed that the expression level of pAKT and total AKT proteins in 5-FU-resistant CRC cells were significantly higher than those in parental CRC cells, suggesting that activation of AKT signaling might be a mechanism involved in the development of 5-FU resistance in CRC cells. Of course, whether there is a direct or indirect correlation between miR-149 downregulation and activation of AKT signaling in 5-FU-resistant CRC cells is unclear and needs to be further elucidated. Then, we analyzed the effects of miR-149 expression on the 5-FU sensitivity of CRC cells by gain- and loss-of-function assays. It could be observed that upregulation of miR-149 could moderately inhibit growth of 5-FU-resistant CRC cells. Meanwhile, re-expression of miR-149 could reverse the 5-FU resistance of 5-FU-resistant CRC cells by enhancing 5 -FU-inducing apoptosis, while knockdown of miR-149 could induce the 5-FU resistance of parental CRC cells by decreasing 5-FU-inducing apoptosis.

FOXM1 (also known as HFH-11, MPP-2, WIN, and Trident), is a typical transcription factor that belongs to the Forkhead Box family, which is evolutionarily conserved and is defined by having a common DNA-binding domain called Forkhead or winged-helix domain [26]. The overexpression of FOXM1 and other forkhead transcription factors has been reported to play important roles in tumor development [27]. Previously, we have shown that FOXM1 overexpression is a molecular marker predicting increased invasive / metastatic potential of CRC and a poorer prognosis [12]. Furthermore, we identified that FOXM1 is a

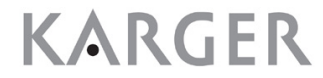


direct and functional target of miR-149 in CRC and that dysregulation of miR-149/FOXM1 correlates with CRC migration and invasion [11]. Recently, it has been reported that FOXM1 can affect chemosensitivity in a variety of human cancers, including ovarian cancer, gastric cancer, breast cancer and lung cancer [28-31]. In the present study, we first testified that miR-149 could regulate the sensitivity of CRC cells to 5-FU by directly targeting FOXM1. This conclusion was based on our several experimental results. Herein, first, the luciferase activity assay indicated that miR-149 could bind to the 3'-UTR sequence of FOXM1 mRNA in 5-FUresistant CRC cells. Second, upregulation of miR-149 decreased the expressions of FOXM1 mRNA and protein in 5-FU-resistant CRC cells, while downregulation of miR-149 increased the expressions of FOXM1 mRNA and protein in parental CRC cells. Third, functional assay indicated that silencing of FOXM1 could mimic the effects of miR-149 upregulation on the 5-FU sensitivity of CRC cells. Finally, the expression level of miR-149 was higher in the 5-FUresponding CRC tissues compared to the non-responding tissues, and inversely correlated with FOXM1 mRNA expression. Since the size of tissue sample in this study is small, further investigation of a larger patient population will be necessary to confirm the correlation of miR-149 expression with the responses of CRC patients to 5-FU-based chemotherapy.

Taken together, reduced miR-149 is a critical factor in the mechanisms by which CRC cells resist the cytotoxicity of 5-FU. Also, re-expression of miR-149 could increase the 5-FU sensitivity of CRC cells via enhancing 5-FU-inducing apoptosis by targeting FOXM1. Therefore, targeted therapies to miR-149/FOXM1 signaling may increase sensitivity to 5-FU treatment and may promise a therapeutic strategy for 5-FU-resistant CRC.

\section{Acknowledgements}

This work was supported by grants from the National Natural Science Foundation of China (No.81272394). Thanks to every one of the Department of Surgery or Pathology in Jingling Hospital for their sincere help and technique support.

\section{Disclosure Statement}

No potential conflicts of interest were disclosed

\section{References}

1 Siegel R, Ma J, Zou Z, Jemal A: Cancer statistics, 2014. CA Cancer J Clin 2014;64:9-29.

2 Longley DB, Harkin DP, Johnston PG: 5-fluorouracil: mechanisms of action and clinical strategies. Nat Rev Cancer 2003;3:330-338.

3 Longley DB, Allen WL, Johnston PG: Drug resistance, predictive markers and pharmacogenomics in colorectal cancer. Biochim Biophys Acta 2006;1766:184-196.

4 Moreno-Moya JM, Vilella F, Simón C: MicroRNA: key gene expression regulators. Fertil Steril 2014;101:1516-1523.

5 Jovanovic M, Hengartner MO: miRNAs and apoptosis: RNAs to die for. Oncogene 2006;25:6176-6187.

6 Miska EA: How microRNAs control cell division, differentiation and death. Curr Opin Genet Dev 2005;15:563-568.

7 Magee P, Shi L, Garofalo M: Role of microRNAs in chemoresistance. Ann Transl Med 2015;3:332.

8 Cellini F, Morganti AG, Genovesi D, Silvestris N, Valentini V: Role of microRNA in response to ionizing radiations: evidences and potential impact on clinical practice for radiotherapy. Molecules 2014;19:53795401.

9 Zhang W, Zou C, Pan L, Xu Y, Qi W, Ma G, Hou Y, Jiang P: MicroRNA-140-5p inhibits the progression of colorectal cancer by targeting VEGFA. Cell Physiol Biochem 2015;37:1123-1133.

10 Fang Y, Sun B, Xiang J, Chen Z. MiR-301a promotes colorectal cancer cell growth and invasion by directly targeting SOCS6. Cell Physiol Biochem 2015;35:227-236. 


\section{Cellular Physiology Cell Physiol Biochem 2016;39:617-629 \begin{tabular}{l|l|l} 
and BOI: 10.1159/000445653 & $\begin{array}{l}\text { ( ) 2016 The Author(s). Published by S. Karger AG, Basel } \\
\text { www.karger.com/cpb }\end{array}$
\end{tabular}}

Liu et al.: MicroRNA-149 and 5-FU Resistance in Colorectal Cancer

11 Xu K, Liu X, Mao X, Xue L, Wang R, Chen L, Chu X: MicroRNA-149 suppresses colorectal cancer cell migration and invasion by directly targeting forkhead box transcription factor FOXM1. Cell Physiol Biochem 2015;35:499-515.

12 Chu XY, Zhu ZM, Chen LB, Wang JH, Su QS, Yang JR, Lin Y, Xue LJ, Liu XB, Mo XB: FOXM1 expression correlates with tumor invasion and a poor prognosis of colorectal cancer. Acta Histochem 2012;114:755762.

13 Li L, Liu T, Li Z, Zhang L, Zhang Z: The miR-149 rs2292832 T/C polymorphism may decrease digestive cancer susceptibility: an updated meta-analysis. Int J Clin Exp Med 2015;8:15351-15361.

14 Wei WJ, Lu ZW, Li DS, Wang Y, Zhu YX, Wang ZY, Wu Y, Wang YL, Ji QH: Association of the miR-149 Rs2292832 polymorphism with papillary thyroid cancer risk and clinicopathologic characteristics in a Chinese population. Int J Mol Sci 2014;15:20968-20981.

15 Huang GL, Lu Y, Pu XX, He YX, Chen ML, Li YZ, Tang SY, Che H, He Z: Association study between miR-149 gene polymorphism and nasopharyngeal carcinoma. Biomed Rep 2013;1:599-603.

16 Zhang Y, Guo X, Xiong L, Yu L, Li Z, Guo Q, Li Z, Li B, Lin N: Comprehensive analysis of microRNA-regulated protein interaction network reveals the tumor suppressive role of microRNA-149 in human hepatocellular carcinoma via targeting AKT-mTOR pathway. Mol Cancer 2014;13:253.

17 Wang Y, Zheng X, Zhang Z, Zhou J, Zhao G, Yang J, Xia L, Wang R, Cai X, Hu H, Zhu C, Nie Y, Wu K, Zhang D, Fan D: MicroRNA-149 inhibits proliferation and cell cycle progression through the targeting of ZBTB2 in human gastric cancer. PLoS One 2012;7:e41693.

18 Pan SJ, Zhan SK, Pei BG, Sun QF, Bian LG, Sun BM: MicroRNA-149 inhibits proliferation and invasion of glioma cells via blockade of AKT1 signaling. Int J Immunopathol Pharmacol 2012;25:871-881.

19 Chan SH, Huang WC, Chang JW, Chang KJ, Kuo WH, Wang MY, Lin KY, Uen YH4, Hou MF, Lin CM, Jang TH, Tu CW, Lee YR, Lee YH, Tien MT, Wang LH: MicroRNA-149 targets GIT1 to suppress integrin signaling and breast cancer metastasis. Oncogene 2014;33:4496-4507.

20 He DX, Gu XT, Li YR, Jiang L, Jin J, Ma X: Methylation-regulated miR-149 modulates chemoresistance by targeting GlcNAc N-deacetylase/N-sulfotransferase-1 in human breast cancer. FEBS J 2014;281:4718-4730.

21 She X, Yu Z, Cui Y, Lei Q, Wang Z, Xu G, Xiang J, Wu M, Li G: miR-128 and miR-149 enhance the chemosensitivity of temozolomide by Rap1B-mediated cytoskeletal remodeling in glioblastoma. Oncol Rep 2014;32:957-964.

22 Li PL, Zhang X, Wang LL, Du LT, Yang YM, Li J, Wang CX: MicroRNA-218 is a prognostic indicator in colorectal cancer and enhances 5-fluorouracil-induced apoptosis by targeting BIRC5. Carcinogenesis 2015;36:1484-1493.

23 He J, Xie G, Tong J, Peng Y, Huang H, Li J, Wang N, Liang H: Overexpression of microRNA-122 re-sensitizes 5-FU-resistant colon cancer cells to 5-FU through the inhibition of PKM2 in vitro and in vivo. Cell Biochem Biophys 2014;70:1343-1350.

24 Zhang Y, Talmon G, Wang J: MicroRNA-587 antagonizes 5-FU-induced apoptosis and confers drug resistance by regulating PPP2R1B expression in colorectal cancer. Cell Death Dis 2015;6:e1845.

25 Nishida N, Yamashita S, Mimori K, Sudo T, Tanaka F, Shibata K, Yamamoto H, Ishii H, Doki Y, Mori M: MicroRNA-10b is a prognostic indicator in colorectal cancer and confers resistance to the chemotherapeutic agent 5-fluorouracil in colorectal cancer cells. Ann Surg Oncol 2012;19:3065-3071.

26 Katoh M, Katoh M: Human FOX gene family (Review). Int J Oncol 2004;25:1495-1500.

27 Halasi M, Gartel AL: Targeting FOXM1 in cancer. Biochem Pharmacol 2013;85:644-652.

28 Zhao F, Siu MK, Jiang L, Tam KF, Ngan HY, Le XF, Wong OG, Wong ES, Gomes AR, Bella L, Khongkow P, Lam EW, Cheung AN: Overexpression of forkhead box protein M1 (FOXM1) in ovarian cancer correlates with poor patient survival and contributes to paclitaxel resistance. PLoS One2014; 9:e113478.

29 Li X, Yao R, Yue L, Qiu W, Qi W, Liu S, Yao Y, Liang J: FOXM1 mediates resistance to docetaxel in gastric cancer via up-regulating Stathmin. J Cell Mol Med 2014;18:811-823.

30 Nestal de Moraes G, Delbue D, Silva KL, Robaina MC, Khongkow P, Gomes AR, Zona S, Crocamo S, Mencalha AL, Magalhães LM, Lam EW, Maia RC: FOXM1 targets XIAP and Survivin to modulate breast cancer survival and chemoresistance. Cell Signal 2015;27:2496-2505.

31 Liu Y, Chen X, Gu Y, Zhu L, Qian Y, Pei D, Zhang W, Shu Y: FOXM1 overexpression is associated with cisplatin resistance in non-small cell lung cancer and mediates sensitivity to cisplatin in A549 cells via the JNK/ mitochondrial pathway. Neoplasma 2015;62:61-71. 